Documentation et bibliothèques

DOCUMENTATION BIBLIOTHEQUES

\title{
Analyse opérationnelle dans les bibliothèques de l'université de Sherbrooke
}

\section{Trefflé Michaud}

Volume 21, numéro 1, mars 1975

URI : https://id.erudit.org/iderudit/1055518ar

DOI : https://doi.org/10.7202/1055518ar

Aller au sommaire du numéro

\section{Éditeur(s)}

Association pour l'avancement des sciences et des techniques de la documentation (ASTED)

\section{ISSN}

0315-2340 (imprimé)

2291-8949 (numérique)

Découvrir la revue

Citer cet article

Michaud, T. (1975). Analyse opérationnelle dans les bibliothèques de l'université de Sherbrooke. Documentation et bibliothèques, 21(1), 41-47. https://doi.org/10.7202/1055518ar
Résumé de l'article

L’opération " Analyse opérationnelle " représente une partie seulement d'une entreprise plus vaste dénommée " Analyse des systèmes ", travail qui comprend également ce qu'on appelle l'« Analyse budgétaire ». Commencée il y a trois ans (septembre 1971) dans les bibliothèques de l'université de Sherbrooke, l'analyse opérationnelle, malgré de nombreuses complications de toute nature, permet désormais des économies annuelles et répétitives d'environ $\$ 420,000$ (à peu près un tiers de la masse salariale). Il est nécessaire maintenant de compléter la formation du personnel de cadre pour maintenir, et améliorer si possible, les gains et les résultats obtenus.
Tous droits réservés (c) Association pour l'avancement des sciences et des techniques de la documentation (ASTED), 1975
Ce document est protégé par la loi sur le droit d'auteur. L'utilisation des services d'Érudit (y compris la reproduction) est assujettie à sa politique d'utilisation que vous pouvez consulter en ligne.

https://apropos.erudit.org/fr/usagers/politique-dutilisation/ 


\section{Analyse opérationnelle dans les bibliothèques de l'université de Sherbrooke}

Trefflé Michaud

Directeur des bibliothèques

Université de Sherbrooke

L'opération "Analyse opérationnelle» représente une partie seulement d'une entreprise plus vaste dénommée "Analyse des systèmes», travail qui comprend également ce qu'on appelle l' "Analyse budgétaire". Commencée il y a trois ans (septembre 1971) dans les bibliothèques de l'université de Sherbrooke, l'analyse opérationnelle, malgré de nombreuses complications de toute nature, permet désormais des économies annuelles et répétitives d'environ $\$ 420,000$ (à peu près un tiers de la masse salariale). Il est nécessaire maintenant de compléter la formation du personnel de cadre pour maintenir, et améliorer si possible, les gains et les résultats obtenus.

The process of "operations analysis" is only a part of a larger undertaking called "systems analysis", which includes as well the "budget analysis". In spite of diverse complications, the operations analysis, which started three years ago (in September 1971) in the libraries of Sherbrooke University, allows from this point on annual and repetitive savings of about $\$ 420,000$ (nearly a third of the wages bill). It is necessary at this time to complete the formation of the executive staff in order to maintain, and to improve, if possible, these gains.

El proceso de "análisis operacional» representa sólo una parte de una empresa más amplia llamada "análisis de los sistemas", trabajo que incluye también el "análisis de los presupuestos». Empezado hace tres años (en septiembre 1971) en las bibliotecas de la Universidad de Sherbrooke, el análisis operacional, a pesar de varias complicaciones, permite realizar economias anuales de unos $\$ 420,000$ (casi la tercera parte de los gastos salariales). Es preciso ahora completar la formación del personal de dirección para mantener y mejorar, si es posible, las ganancias y los resultados obtenidos.

\section{Définition théorique du concept d'analyse opérationnelle}

L'analyse opérationnelle, comme l'analyse budgétaire et, plus généralement, l'analyse des systèmes, est une opération administrative qui ne vise qu'un seul objectif: obtenir le rendement maximal possible (en y incluant, bien sûr, le facteur humain) à partir d'un investissement minimal des ressources d'un système.

L'analyse des systèmes (compagnies, industries, bibliothèques, etc.) comporte un ensemble d'étapes que l'on ne traitera que sommairement, vu l'ampleur du sujet.

\section{1 - Examen des économies ou gains possibles dans chaque secteur des ressources d'un système}

Afin d'obtenir un rendement maximum dès le début d'une entreprise aussi vaste que celle de l'analyse d'un système, il faut d'abord examiner les secteurs susceptibles de conduire, dans un premier temps, aux économies et aux améliorations les plus importantes; en somme, il s'agit de déceler les secteurs prioritaires et de s'y attaquer dès le début.

Pour ce faire, il faut se livrer à l'exercice de l'analyse budgétaire. Grâce aux services d'un spécialiste de l'administration (et du 
système analysé si possible), il faut étudier:

- les coûts par rapport aux produits obtenus;

- l'évolution des budgets, i.e. sources et augmentations des revenus par rapport aux augmentations incompressibles des dépenses (par exemple, celles dues à l'inflation, etc.).

Au fur et à mesure du déroulement de cette opération, l'on découvre assez rapidement que la technique de l'analyse des coûts par service (P.P.B.S.) devient un outil indispensable pour déterminer avec précision, et éventuellement corriger, les coûts prohibitifs.

II faut donc examiner successivement les cinq postes budgétaires généraux suivants qui revêtent une importance variable selon les organisations en cause; l'ordre d'importance présenté ici s'applique fort bien au budget d'une bibliothèque.

a - Salaires: quelle proportion du budget général est en cause? Quelle partie de la masse salariale est consacrée à chaque catégorie de personnel? Quel est le rythme annuel d'augmentation des salaires?

$b$-Collections: sont-elles dédoublées sur place et dans la région? II faut alors définir les juridictions et coordonner les secteurs au niveau de la sélection. Sont-elles trop exhaustives: objectifs trop éloignés ou trop vastes? II faut alors analyser les divers besoins plus ou moins immédiats des cours et des programmes de recherche. Sont-elles mal distribuées? II faut alors recourir à des formules de distribution utilisant des critères appropriés.

II importe de noter ici que l'analyse et la planification du développement des collections ne peuvent être efficaces sans l'aide de statistiques de toute nature qu'il semble assez difficile, sinon impossible, d'obtenir sans l'automatisation du bureau de prêt tout au moins.

$c$ - Fournitures de bureau, équipement et espaces: les gains possibles à ces postes budgétaires peuvent facilement découler de gains obtenus au chapitre des salaires et des collections.

Il est toutefois possible d'obtenir des gains directs, si l'on élabore, au départ, des normes qui respectent le caractère fonctionnel du travail à accomplir et des services à rendre, même sans procéder à aucune analyse ultérieure des opérations et des collections.

\section{2 - Déroulement de l'analyse générale d'un système}

Avant de réaliser l'analyse opérationnelle elle-même, dans les secteurs reconnus comme prioritaires (normalement celui des salaires), il importe de:

a) réviser les objectifs du système: qualité et quantité des usagers, des cours et des programmes de recherche, mode d'utilisation des ressources par les usagers. Une enquête sur les services offerts à l'usager s'imposera alors, pour identifier les principales tendances en matière de besoins;

b) examiner les structures du système: il peut en effet arriver que de mauvaises structures paralysent les efforts d'une foule de personnes pourtant qualifiées. Un tel examen permettra de confirmer, en cas de lacunes réelles, l'existence de gains possibles;

c) examiner les qualifications du personnel, surtout le personnel de cadre: toute organisation en effet n'est-elle pas à l'image de ses cadres?

Toutes ces analyses devraient, si possible, se réaliser grâce aux techniques de M.B.O. (Management by Objectives) et à un travail d'équipe. Au sein d'un organisme ou d'un système, même le plus modeste, personne, en effet, ne peut prétendre remplir les fonctions de tous les autres membres de l'équipe; il faut donc apprendre à s'intégrer dans un groupe, à déléguer le plus possible ses fonctions, à établir des consensus, à motiver le personnel, etc.

\section{3 - Formation du personnel profession- nel (en commençant par les cadres)}

S'il est relativement facile de réviser et de corriger les objectifs d'un système ainsi que ses structures, l'on ne peut toutefois se lancer dans l'analyse opérationnelle des secteurs prioritaires (surtout celui des salaires) sans penser à la formation du personnel professionnel, notamment celui des cadres. Ceux-ci doivent être familiarisés avec les cinq grandes fonctions de l'administration mais aussi avec les techniques de l'analyse des systèmes et de l'analyse opérationnelle. Ceci pourra se faire à l'aide de cours, de séminaires, d'expériences-pilotes, etc.

a - Les cinq grandes fonctions de l'administration (par ordre de priorité interne):

- planifier: étudier et fixer les objectifs, les 
politiques et les programmes d'action à court, moyen et long termes, etc.;

- organiser: définir les structures, les unités de fonctionnement et les relations «inter-groupes», etc.;

- mobiliser les ressources: personnel, espaces, fournitures, etc.;

- diriger: conduire quotidiennement les opérations, en tendant vers la décision collégiale et en prévenant les actes d'insubordination qui provoquent irrémédiablement du gaspillage sous toutes ses formes possibles; il faut donc donner les instructions appropriées, motiver, coordonner, favoriser les bonnes relations, etc.;

- contrôler: vérifier dans quelle mesure les résultats, visés par l'entreprise au niveau des individus, des secteurs, etc., ont été atteints tant quantitativement que qualitativement.

II est à noter que cette énumération des fonctions administratives est présentée par ordre décroissant d'importance hiérarchique; le bon administrateur doit d'abord planifier, ensuite organiser, s'il lui reste du temps disponible, etc. Plar ailleurs, le bon chef d'équipe (supérieur immédiał) doit d'abord contrôler, diriger, etc. Les données recueillies deviennent des éléments de base nécessaires à la planification à un niveau plus élevé de la direction; elles seront également nécessaires à l'évaluation du personnel.

$b$ - Les techniques de. l'analyse des systèmes et de l'analyse opérationnelle.

L'opération "Analyse opérationnelle" consiste principalement à colliger, dans des tableaux quotidiens et mensuels, l'ensemble des opérations selon le temps ou la fréquence. Ensuite, il importe de se poser, sur les dites opérations, cinq questions fondamentales: pourquoi? par qui? comment? où? quand? ceci afin d'obtenir une plus grande efficacité, i.e. le meilleur compromis possible entre la quantité, la qualité et les coûts des opérations. ${ }^{1}$

Lorsque le secteur important à analyser s'avère celui des salaires, l'analyse dite opérationnelle doit principalement porter sur les aspects suivants, en commançant, bien en-

1. Pour plus de détails, il suffira de consulter un article déjà publié sur cette question: Trefflé Michaud, "Étapes à franchir dans la réalisation de tout projet ou opération », in L'Administration budgétaire de la bibliothèque, Montréal, ACBLF, 1973,53 et 55-62. tendu, par les opérations les plus nombreuses ou les plus coûteuses:

- pertes de temps: ponctualité et discipline, pendant les heures de travail (spécialement au début ou à la fin), surtout dans les secteurs de grande concentration d'employés;

- opérations: dont les objectifs sont partiellement ou totalement inutiles; qui font double emploi, donc inutiles; mal coordonnées, donc nécessitant plus de temps de réalisation; réalisées aux mauvais endroits (déplacements inutiles); réalisées aux mauvais moments, donc pouvant impliquer trop de déplacement et l'absence de toute quantification (batch processing); impliquant des méthodes inefficaces ou entraînant des durées plus longues de réalisation; réalisées par des personnes "sous-qualifiées" (rendements moindres en quantité et en qualité) ou au contraire par des personnes "surqualifiées" (coûts inutiles).

\section{4 - Réalisation de l'analyse opération- nelle}

Une fois toutes les étapes précédentes accomplies, l'on peut alors se lancer dans l'entreprise souvent tumultueuse mais combien stimulante de l'analyse opérationnelle ellemême.

II ne faut toutefois pas oublier que, sans mettre de côté les cinq grandes fonctions administratives, certaines conditions permettront d'assurer une meilleure réussite de l'analyse d'un système et de l'analyse opérationnelle; ces conditions pourront, a fortiori, contribuer à maintenir les gains et les améliorations obtenues:

a - un analyste avec pouvoir décisionnel: donc placé au plus haut niveau de la ligne hiérarchique si possible, ceci afin de pouvoir contrer efficacement les oppositions irréductibles;

b - un analyste recruté à l'intérieur de l'organisation: la motivation générale ne peut que mieux s'en porter;

c - un analyste qualifié à la fois en bibliothéconomie et en administration; si cette condition ne peut être remplie, il s'avère plus économique et plus rapide de compléter les connaissances administratives d'un bibliothécaire que de procéder à l'inverse; 
d- la ratification complète, par les autorités supérieures, du projet d'analyse avant sa réalisation; une telle ratification évitera les surprises désagréables et garantira les appuis requis aux moments opportuns;

e - la complète collaboration du service du personnel, surtout si le service est externe à l'organisation: conflits, plaintes, griefs, transferts, congédiements;

$f$ - des sessions intensives de sensibilisation et de motivation avant et durant l'analyse; il faut donc étudier tous les phénomènes de résistance au changement, et surtout en tenir compte; il faut aussi procéder avec une certaine discrétion, afin d'éviter les rumeurs génératrices d'insécurité et de résistance; il faut même, au besoin, éviter certains mots ou appellations qui engendrent la panique, par exemple l'appellation "analyse opérationnelle» elle-même;

\section{$g$ - le recours constant à du travail d'équipe:}

- utiliser les techniques de M.B.O.;

- mettre en œuvre les techniques administratives, les techniques de la motivation et de la communication, etc.;

- sélectionner rigoureusement le nouveau personnel requis, notamment celui de cadre;

- se résigner à démettre (voire même à congédier) un cadre (ou tout membre du personnel en général) qui ne peut se qualifier comme administrateur ou qui est insoumis. En effet, pour savoir commander, il faut être capable de se soumettre en dernier ressort, sinon l'équipe unique ne peut exister, ce qui compromet le fonctionnement efficace d'une organisation ou d'un système; mieux vaut un cadre de valeur moyenne, mais intégré, qu'une vedette qui fait bande à part ou cavalier seul;

$h$ - réinvestissement des gains pour améliorer: le système (création possible de nouveaux services) et les traitements du personnel de cadre, des professionnels et autres.

\section{5 - Contraintes pouvant entraver partiel- lement ou totalement la réalisation d'une analyse opérationnelle:}

a - la nécessité d'œuvrer avec un syndicat accrédité ou non, ou encore des délais très courts avant une demande d'accréditation d'un syndicat; b - l'impossibilité d'obtenir les fonds nécessaires pour payer les analystes requis;

c - la difficulté de déplacer ou de congédier le personnel inefficace: le seul remède possible devient alors le roulement (turn over) du personnel.

\section{Description des faits de l'analyse opérationnelle menée dans les bibliothèques de l'université de Sherbrooke}

\section{1 - Objectifs}

Au départ, il n'apparaissait pas sage de remettre en cause les objectifs généraux du système; il a semblé préférable, en effet, grâce à une analyse budgétaire simplifiée, d'essayer de réaliser le plus rapidement possible des économies dans les secteurs aux dépenses importantes, soit le secteur des collections (25\%) et le secteur des salaires $(70 \%)$.

Après, il devenait relativement facile, à l'aide des fonds disponibles, d'améliorer nos services ou d'en créer d'autres, quitte alors à remettre partiellement ou totalement en cause les objectifs du système. Pour ce faire, le conseil d'administration de l'université avait décidé de permettre le virement de toutes les économies réalisées, à l'amélioration principalement du budget des collections.

\section{2-Bref historique}

Afin de ne pas allonger le texte, l'historique qui suit est présenté sous forme schématique et représente la partie du programme réalisée jusqu'à ce jour:

- juillet 1971:

- présentation des documents de base: a) responsabilités des directeurs;

b) nomination d'adjoints aux directeurs:

c) tableaux d'opérations;

- présentation du programme général d'action:

a) bibliothécaires: expérimentation des tableaux d'opérations du 1er septembre au 15 octobre 1971 puis mise en application;

b) personnel technique et de bureau: expérimentation des tableaux du 15 octobre au 23 décembre 1971 et mise en application par la suite. 
- fin novembre 1971:

- suspension de l'analyse opérationnelle, vu la demande d'accréditation du syndicat des employés de soutien.

- mars à mai 1972:

- préparation d'un schème théorique d'opérations restructurées pour chaque service. Ce schème fut préparé par 3 ou 4 analystes, sans aucun autre apport durant cette période de demande d'accréditation syndicale.

- juin 1972:

- création d'un nouveau service: le Service bibliographique;

- début de l'implantation de l'analyse opérationnelle à la bibliothèque générale; la première étape de cette implantation est terminée en avril 1973.

- septembre 1972:

- implantation de l'analyse opérationnelle au service des acquisitions; la première étape de cette opération est terminée en mars 1973;

- implantation de l'analyse à la bibliothèque des sciences; première étape terminée en décembre 1972.

- janvier 1973:

- implantation de l'analyse à la bibliothèque médicale; première étape terminée en septembre 1973.

- mars 1973:

- implantation de l'analyse au service du catalogage; en septembre 1973, la première et la seconde étape étaient terminées;

- implantation de l'analyse à la bibliothèque de droit; en mai 1974, la première étape était terminée.

- janvier à mars 1974:

- fin de la seconde et dernière étape au Service bibliographique et au Service des acquisitions, et enfin de la troisième et dernière étape au Service du catalogage.

- mars 1974:

- création du secteur Services techniques et mise en place de son directeur.

- septembre à décembre 1974:

- deuxième et dernière étape à la bibliothèque générale.
3 - Résumé des divers événements syndicaux

- 2 décembre 1971:

- demande d'accréditation du syndicat des employés de soutien de la bibliothèque (personnel technique et de bureau).

-4 juillet 1972

- refus de la demande d'accréditation par le commissaire-enquêteur, à cause du caractère restreint de l'unité de négociation qu'il désire voir s'étendre à tout le personnel de soutien (technique, bureau et ouvrier) de l'université de Sherbrooke, à la requête de celle-ci.

-12 juillet 1972:

- la cause est portée en appel.

-12 avril 1973:

- maintien du refus de la demande d'accréditation.

-17 avril 1973:

- formation d'un nouveau syndicat visant à regrouper cette fois tout le personnel de soutien de l'université de Sherbrooke (SPSUS).

- 17 juin 1974:

- demande d'accréditation du SPSUS.

- 22 août 1974:

- accréditation officielle du SPSUS.

- décembre 1971 à août 1974:

- le syndicat soumet 16 plaintes au commissaire-enquêteur; 14 de celles-ci sont soit perdues, soit retirées par le syndicat, les deux autres sont réglées hors cours à la requête du syndicat.

\section{4 - Autres difficultés rencontrées}

L'opération "Analyse opérationnelle» a suscité, avec une fréquence constante, de nombreux autres problèmes plus ou moins graves.

\section{a) Opposition des directeurs en place.}

L'opposition profonde et plus ou moins ouverte de 5 des 6 directeurs en place ne permit pas de lancer l'opération "Analyse opérationnelle" dans des conditions optimales de réussite: 
- impossibilité d'utiliser des techniques de «Management by Objectives (M.B.O.)" et de travailler en équipe;

- absence de formation (cours, séminaires, expériences-pilotes, etc.) du personnel (en commençant par le personnel de cadre) aux cinq grands principes de l'administration ainsi qu'aux techniques de l' «Analyse des systèmes" et de l' "Analyse opérationnelle» elle-même.

Cette opposition provoqua bientôt, avec le concours d'un climat d'insécurité collective, la formation d'un syndicat des employés de soutien dont la demande d'accréditation, à la fin de novembre 1971, bloqua toute l'opération «Analyse opérationnelle» jusqu'en mars 1972.

La même opposition provoqua le départ (dont 1 congédiement) des 5 directeurs en cause.

b) Demandes d'enquête sur l'administration générale du directeur des bibliothèques.

Cinq demandes d'enquête se succédèrent durant une période de 7 mois entre février et août 1972:

- 3 soumises par le syndicat;

- 1 soumise par 3 directeurs dissidents de la bibliothèque;

- 1 présentée par 5 professeurs en désaccord avec la sélection des documents, soi-disant irrationnelle, pratiquée à la bibliothèque.

Aucune de ces demandes d'enquête ne s'avéra fondée; mais chacune contribua grandement à créer tension, insécurité, discorde et imbroglios complètement inutiles, tout cela conséquence soit du changement lui-même, soit de la résistance au changement.

c) Manque de formation du personnel professionnel sur place (notamment celui de cadre)

La nomination de jeunes bibliothécaires inexpérimentés à des postes de cadre, suite au départ de 5 des 6 directeurs en place, de même que la nécessité d'un changement assez radical des méthodes de gestion et de contrôle, ont continuellement mis en évidence la nécessité et d'informer et de former la grande majorité des bibliothécaires sur les thèmes mentionnés dans les paragraphes précédents.
Voilà pourquoi, depuis plus d'un an déjà la bibliothèque a participé et même mis sur pied, dans bon nombre de cas, une série d'activités d'information et de formation aux fonctions professionnelles: entraînement au travail en équipe, gestion du personnel, analyse opérationnelle, relations humaines et leadership, administration générale, fondements psychologiques des relations interpersonnelles, etc.

\section{5 - Résultats obtenus}

Si l'on confronte les aspects positifs et négatifs, il semble bien que cette opération valait la peine d'être conduite, et vaut toujours la peine d'être mise à jour. Les efforts constants et répétés, ainsi que les nombreux problèmes à surmonter sont nettement contrebalancés par les résultats obtenus:

- maintien au même niveau, depuis 1971 du budget de fonctionnement, grâce à une meilleure efficacité technique et administrative; seule est venue s'ajouter l'indexation au coût de la vie: 8 à $9 \%$ au chapitre des salaires et $3 \%$ pour les autres dépenses;

- économies répétitives et annuelles désormais de l'ordre de $\$ 420,000$, grâce à une contraction et réduction totales de 65 employés (11 bibliothécaires, 8 techniciens et 46 commis) si l'on inclut les quelque 24 nouveaux postes requis par les directeurs en place au début de 1971 et refusés par les instances supérieures de l'université. Si l'on exclut ces quelque 24 nouveaux postes, la contraction se chiffre à environ 40 employés ( 33 commis, 1 technicien et 6 bibliothécaires) dont 4 ( 1 bibliothécaire, 1 technicien et 2 commis) ont été affectés à de nouveaux services;

- investissement d'une partie des économies pour améliorer le budget des collections et ainsi constamment lutter contre la perte de son pouvoir d'achat suite aux effets dévastateurs de l'inflation. Une somme additionnelle de $\$ 180$, $336.60 \mathrm{a}$, de la sorte, été injectée dans le budget des collections depuis les trois dernières années (1971-74);

- investissement du reste des économies:

a) dans la création de nouveaux services (création du Centre de documentation de la bibliothèque (1 bibliothécaire et 1 bibliotechnicien) et mise en place d'un Service bibliographique 
pour des fins de référence, acquisition et catalogage);

b) dans l'amélioration des services existants (installation de deux systèmes anti-vol aux bibliothèques générale et médicale; achat d'un duplicateur à cassette et des cassettes requises; projet d'automatisation du prêt et d'informatisation des index alphabétique et systématique du catalogue systématique $(36,000$ indices L.C.); participation à l'expérience de télécatalogage coopératif des universités du Québec et de I'Ontario; etc.);

c) dans l'augmentation du traitement des employés les plus méritants: employés de soutien et bibliothécaires.

\section{6 - Ce qui reste à compléter}

De janvier à juin 1975, il faudra terminer la seconde et dernière étape de l'opération "Analyse opérationnelle" dans les bibliothèques de droit, médecine et sciences, tout en coordonnant le plus possible les schèmes d'opération de ces trois bibliothèques avec ceux de la bibliothèque générale.

II faudra également continuer la formation du personnel de cadre si l'on veut maintenir, et améliorer si possible, les résultats obtenus jusqu'à présent; ainsi, la délégation d'autorité requise pourra s' effectuer et la succession aux postes de cadre se préparer.

Il faudra enfin aller de l'avant dans l'automatisation de nos opérations, celles-ci étant préalablement analysées et coordonnées. Sans ce travail préliminaire, il demeure en effet illusoire et très coûteux de tenter d'automatiser des opérations: même si l'ordinateur possède une mémoire prodigieuse qui opère à des vitesses fantastiques, celui-ci ne raisonne qu'en autant que l'homme réussit à lui prêter les opérations de sa propre intelligence. Par ailleurs, les coûts de l'informatique documentaire accusent maintenant une constante tendance à la baisse.

\section{Conclusion}

Nous avons dû, au cours des trois dernières années, tenter de relever un certain nombre de défis de taille; les opérations "Analyses budgétaire, opérationnelle et de systèmes» ont permis de rationaliser et de rendre plus efficace et moins dispendieux le fonctionnement de la bibliothèque.
Tout ne s'est pas déroulé sans heurts ni sans problèmes, principalement au niveau des relations de travail avec le personnel en général. Avec l'expérience acquise et le recul du temps, un certain nombre d'écueils auraient pu être contournés et des dégâts inutiles auraient pu être réduits ou même évités. Toutefois, nous pourrons désormais mettre à profit le bagage acquis pour consolider la besogne accomplie jusqu'à maintenant et pour la mener à terme, notamment du côté du travail en équipe et de la formation du personnel.

Bref, si les trois dernières années se sont écoulées sous le signe de l'analyse opérationnelle et de la rationalisation, les deux années à venir seront celles du complément de la formation du personnel de cadre et de l'automatisation, toujours en vue d'une efficacité accrue, tant sur le plan des coûts que de la qualité et de la quantité des services à offrir.

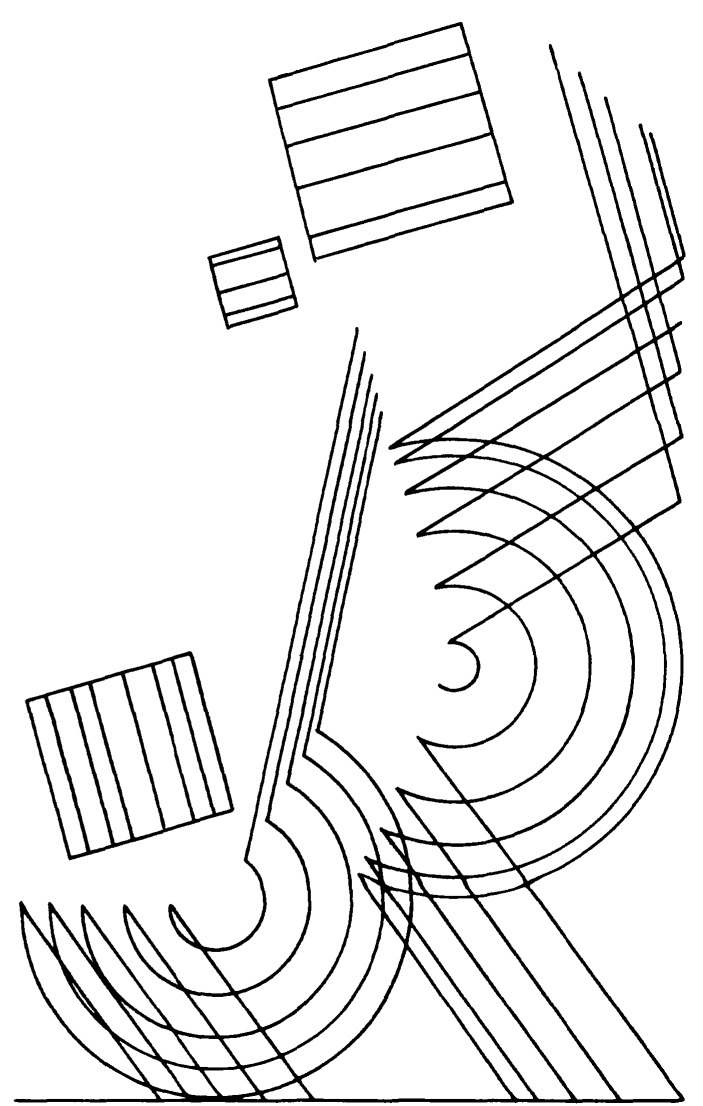

